\title{
Influencia de la transglutaminasa en el rendimiento de la producción de queso Dambo uruguayo
}

\author{
Influence of transglutaminase on the yield \\ of Uruguayan Dambo cheese
}

Escobar, Daniela (1), Arcia, Patrícia (1), Curutchet, Ana (2), Pelaggio, Ronny (1), Urrestarazu, Pedro (1), Márquez, Rosa (1).

(1) Gerencia de I+D+i, Laboratorio Tecnológico del Uruguay, LATU, Uruguay - (2) Gerencia de Análisis, Ensayos y Metrología, Laboratorio Tecnológico del Uruguay, LATU, Uruguay.

Contacto: rmarquez@latu.org.uy

RECIBIDO: 30/05/2014 - APROBADO: 07/11/2014

\begin{abstract}
Resumen
El queso Dambo uruguayo es un queso de mediana humedad de origen danés con formulación modificada. Esta investigación estudió el uso de la enzima transglutaminasa en la producción de queso Dambo tipo barra para aumentar el rendimiento del proceso.

Se fabricaron quesos aplicando un diseño experimental completamente al azar con parcelas divididas medidas en el tiempo, considerando dos factores (concentración de la enzima y momento de agregado) en tres niveles, por triplicado, tomando como referencia el queso sin enzima.

Los quesos fueron analizados a lo largo de cuatro meses en peso, $\mathrm{pH}$, contenido de proteínas, humedad y materia grasa. Se calculó el rendimiento relativo al queso referencia, la recuperación de extracto seco y el contenido en proteínas y materia grasa en el queso respecto a la leche. Se analizó el perfil de textura y se determinaron las propiedades sensoriales con un panel de jueces entrenados.

Los resultados mostraron que la única condición con diferencia significativa al 5\% en el rendimiento relativo fue el momento de agregado de la enzima, resultando como momento óptimo la incorporación de la misma junto con el cuajo, independientemente de la dosis utilizada. El rendimiento logrado fue un $6 \%$ superior al queso referencia. Palabras clave: Queso Dambo tipo barra, enzima, rendimiento, textura, análisis sensorial.
\end{abstract}

\begin{abstract}
The Uruguayan Dambo cheese is a mid-moisture Danish cheese made with a modified formula. This study examined the use of transglutaminase to increase the process yield in the production of Dambo cheese.

Cheeses were made using an experimental design with completely randomized split plot measures over time, considering two factors (enzyme concentration and moment of addition) in three levels, by triplicate, taking as reference the cheese without enzyme.

Cheeses were analyzed along four months considering weight, $\mathrm{pH}$, protein, moisture and fat matter. The yield relative to the reference cheese was calculated, as also the dry matter recovery and protein and fat content in the cheese compared to milk. Texture profile analysis was performed and sensory properties were evaluated using a panel of trained judges.

The results showed that the only condition with a significant difference of $5 \%$ in relative yield was the moment of the enzyme's addition showing that the optimal time corresponds to the addition with rennet regardless the dose used. The yield achieved was $6 \%$ higher than the reference cheese.

Keywords: Dambo cheese bar type, enzyme, yield, texture, sensory analysis.
\end{abstract}

\section{Introducción}

El queso Dambo uruguayo es un queso de mediana humedad o pasta semidura - de entre 36,0 y 45,9\% de humedad según la reglamentación MERCOSUR (1994)—, de origen danés, cuya fórmula ha sido modificada en Uruguay. Se elabora a partir de leche de vaca utilizando cultivos lácticos específicos y de coagulación enzimática, con un tiempo de maduración aproximado de 4 semanas.

El queso Dambo es uno de los más exportados por Uruguay. En 2011, el 85\% de los quesos de pasta semidura fabricados en Uruguay fueron exportados y su producción aumentó un $6 \%$ en 2011 y un $83 \%$ en los últimos cinco años (Ministerio de Ganadería Agricultura y Pesca, 2013). 
Más del 50\% de los quesos de pasta semidura que se producen corresponden a quesos tipo barra. El queso Dambo tipo barra es una formulación más reciente que el queso Dambo tradicional que se fabrica con algunas diferencias tecnológicas que permiten producir un queso de mayor dureza y más feteable, tal como los consumidores lo demandan, con mayor estabilidad en el tiempo. La gran producción de este tipo de quesos en el país fundamenta el interés en aumentar el rendimiento en la producción de queso Dambo tipo barra.

La transglutaminasa (TG) es una enzima ampliamente distribuida en la naturaleza, que se encuentra en tejidos animales, plantas y microorganismos. Se caracteriza por entrecruzar proteínas a través de enlaces covalentes, específicamente entre los aminoácidos glutamina y lisina. Este entrecruzamiento tiene efectos sobre las propiedades de las proteínas, la capacidad de gelificación, la estabilidad térmica, la capacidad de retención de agua, etcétera. La TG es utilizada ampliamente en productos de la pesca, surimi, productos cárnicos, pastas secas, productos lácteos y panificados, entre otros. Tiene un gran potencial para mejorar la firmeza, elasticidad, viscosidad, estabilidad al calor y capacidad de retención de agua en alimentos mediante la reacción enzimática (Kuraishi, et al., 2001).

Durante el proceso de transformación de la leche en queso, las caseínas forman una red proteica conocida como coágulo o gel. Son varios los factores que influyen en la formación de esta cuajada, entre ellos $\mathrm{pH}$, concentración de calcio, concentración de caseína, tratamiento térmico utilizado y proteínas presentes. El uso de TG afecta tanto la primera como la segunda etapa de formación del gel (Bönisch, et al., 2008). La TG forma enlaces entre las $\alpha$ - $\beta$ - y $\kappa$-caseínas (Aaltonen, et al., 2014) y además produce entrecruzamientos con la $\beta$-lactoglobulina y la $\alpha$-lactoalbúmina en la cuajada (Cozzolino, et al., 2003; Özrenk, 2006). Estos entrecruzamientos podrían favorecer un aumento del rendimiento quesero, afectando positivamente la rentabilidad del producto.

Varios autores han estudiado el impacto de la TG en las proteínas de la leche y en las propiedades físicas y de textura de los geles resultantes de la acidificación (Bönisch, et al., 2007; Jaros, et al., 2007, 2010). Özer et al. (2012) demostraron que la acción específica de la TG en geles de cuajo se ve afectada por los parámetros del procesamiento, como la temperatura de coagulación, la concentración de TG y el pH de la leche inicial, por lo cual es necesario optimizar estos parámetros antes de realizar la incorporación de TG en la fabricación de quesos.

Otro ingrediente utilizado en la fabricación de quesos es la enzima quimosina, que actúa sobre la $\kappa$-caseína produciendo caseinomacropéptidos (CMP). Es importante tanto el momento de agregado de la quimosina como la temperatura, para que la acción de la TG no enlentezca la liberación de CMP durante la fase primaria de la coagulación (Özer, et al., 2013).

En base al potencial de la TG se diseñó este estudio con el objetivo de conocer si el uso de la enzima como coadyuvante tecnológico es capaz de aumentar el rendimiento en la producción de uno de los quesos más fabricados en Uruguay, el queso Dambo tipo barra, estudiando las condiciones óptimas del proceso y cómo afecta su uso en las características típicas de dicho queso.

\section{Materiales y Métodos}

El queso Dambo fue elaborado a partir de leche cruda de vaca. La leche se analizó a su ingreso a la planta piloto determinándose: $\mathrm{pH}$ ( $\mathrm{pHmetroMettler} \mathrm{Toledo} \mathrm{SevenMulti,} \mathrm{electrodo} \mathrm{com-}$ binado de penetración InLab), acidez titulable (grados Dornic, ISO/TS 22113/IDF RM 204, 2012), porcentaje de proteínas (ISO 8968/IDF 20, 2001), porcentaje de materia grasa (ISO 1211/IDF1, 2010), porcentaje de extracto seco (ISO 6731/IDF $21,2010)$ y densidad por picnometría (AOAC 925.22, 2005).

Para evitar los efectos de las diferencias de cada lote de leche utilizado, con cada lote de leche se fabricaron cuatro lotes de queso: un lote de queso referencia (sin TG) y tres lotes de queso con TG (ACTIVA ${ }^{\oplus}$ YG, Ajinomoto, Francia), según el diseño aleatorio del experimento. De esta manera se fabricaron un total de 27 lotes de queso con tres concentraciones diferentes de TG agregada en tres momentos distintos del proceso.

Cada lote de queso fue elaborado en tina quesera doble O con doble camisa y sistema de agitación y corte mecánico controlado, a partir de $50 \mathrm{~L}$ de leche previamente pasteurizados a $72{ }^{\circ} \mathrm{C}$ durante 15 segundos. Cuando la leche alcanzó los $33^{\circ} \mathrm{C}$ se incorporaron $10 \mathrm{~mL}$ de cloruro de calcio Promilk ${ }^{\oplus}$ al $33 \%$ y fermentos mesófilos y termófilos homofermentativos de acidificación rápida Choozit ${ }^{\circledR}$ RA21 de Danisco, Dupont, en una dosis de 3,125 DCU (Direct Culture Unit) por lote. A los 30 minutos se agregaron $3 \mathrm{~mL}$ de coagulante de quimosina pura Maxiren $^{\oplus}$ dejándolo actuar durante 25 minutos en las mismas condiciones. Luego se cortó la cuajada con cuchilla mecánica hasta obtener un tamaño de grano de maíz y se cocinó hasta llegar a $42^{\circ} \mathrm{C}$. Se drenó la masa hacia un cajón de pre-prensa con red donde se hizo un pre-prensado manual.

Se formaron los quesos en moldes de polipropileno de $1 \mathrm{Kg}$ de capacidad con desuerador y tapa y se prensaron verticalmente durante 3 horas, con rotaciones intermedias. Cuando los quesos alcanzaron un $\mathrm{pH}$ de 5,4 se refrigeraron a $4 \pm 2{ }^{\circ} \mathrm{C}$. A las 16 horas se sumergieron en salmuera a 19 grados Baumé durante 5 horas con volteo a medio tiempo. Se realizó un oreo por 24 horas a $4 \pm$ $2{ }^{\circ} \mathrm{C}$ y se envasaron a vacío en bolsas Maraflex ARG2. Los quesos fueron conservados en cámara de maduración a $8 \pm 2{ }^{\circ} \mathrm{C}$ hasta el momento de su análisis y fueron analizados en sus parámetros fisicoquímicos a tiempo 0 (momento de envasado), 1, 2, 3 y 4 meses de envasados. El perfil de textura y el análisis sensorial se realizaron a los 1, 2, 3 y 4 meses de envasados.

\section{Diseño experimental}

Se elaboró un diseño experimental completamente al azar con parcelas divididas medidas en el tiempo, considerando dos factores (concentración de la enzima y momento de agregado) en tres niveles. Los experimentos se realizaron por triplicado, tomando como referencia el queso sin enzima.

Las concentraciones de TG utilizadas fueron $0,1,0,5$ y 1,0 unidades TG / g de proteína en leche, donde una unidad de la enzima es la cantidad de enzima que cataliza la formación de $1 \mu \mathrm{mol}$ de ácido l-glutámico Ymonohidroxamato en un minuto a pH 6 y $37^{\circ} \mathrm{C}$.

Los momentos de incorporación de TG en el proceso fueron: «con el cuajo», manteniendo las restantes condiciones de fabricación iguales al queso referencia, «con el cuajo en frío» a $15{ }^{\circ} \mathrm{C}$, habiéndose realizado todo el proceso previo también a $15^{\circ} \mathrm{C}, \mathrm{y}$ «al corte», incorporando la enzima luego de alcanzado el tamaño grano de maíz.

\section{Análisis fisicoquímico de los quesos}

Peso: Se utilizó balanza de precisión Shimadzu BX22KH. pH: Se midió con pHmetro Mettler Toledo SevenMulti y electrodo combinado de penetración InLab. 
Humedad, proteínas, materia grasa: Se analizaron con FoodScan ${ }^{\circledast}$ Labtype 78800 Foss, Dinamarca, previamente calibrado según las normas ISO 5534/IDF 004, 2004, ISO 8968/ IDF 20, 2001 e ISO 1735/IDF 005, 2004, respectivamente.

\section{Perfil de textura instrumental}

Se realizó el análisis de perfil de textura (TPA) con texturómetro TA-XT-Plus Texture Analyzer (Stable Micro Systems Ltd., UK). Se determinaron dureza, elasticidad y cohesividad.

\section{Perfil sensorial}

Se analizaron 15 atributos con un panel de 10 jueces entrenados (ISO 22935-1, 2009; Lavanchy, et al., 1993; Montero, et al., 2005): intensidad de olor, firmeza táctil, firmeza en boca, elasticidad, friabilidad, solubilidad, impresión de humedad, adhesividad, intensidad de sabor, dulce, amargo, salado, ácido, picante y retrogusto. Los jueces evaluaron la intensidad de cada atributo usando una escala no estructurada de 0 a 7 . La evaluación se realizó en una sala normalizada según ISO 8589, luego de 1, 2, 3 y 4 meses de maduración de los quesos.

\section{Análisis de datos}

Para estudiar la variabilidad en todos los parámetros analizados en cada tiempo del estudio, se realizó un ANOVA de dos factores con interacción (concentración de enzima y momento de agregado).

Para estudiar el efecto de la concentración de enzima y el tiempo de maduración, se realizó un ANOVA de dos factores con interacción (concentración de enzima y tiempo de maduración) por diseño de parcelas divididas.

Para determinar la diferencia significativa entre muestras se utilizó el test de Tukey $(\alpha \leq 0.05)$. Los análisis se realizaron usando XLSTAT Version 2011 (Addinsoft 1995-2010, France) e Info Stat Statistical Software versión 2011.

\section{Cálculos de rendimiento $\mathrm{y}$ aprovechamiento de los sólidos de queso}

Rendimiento Relativo (\%) $=\frac{\text { peso del queso con TG }}{\text { peso queso referencia }}$

Las recuperaciones de los sólidos del queso se determinaron según:

Recuperación de $=$ peso queso $\mathrm{x}$ extracto seco queso $\times 100$ Extracto Seco (\%) $=\frac{\text { peso leche } \mathrm{x} \text { extracto seco leche }}{\text { pesto }}$

$\begin{aligned} & \text { Recuperación } \\ & \text { de Proteínas }(\%)\end{aligned}=\frac{\text { peso queso } \mathrm{x} \text { proteínas en queso }}{\text { peso leche } \mathrm{x} \text { proteínas leche }} \times 100$

Recuperación de $=$ peso queso $\mathrm{x}$ materia grasa queso $\times 100$

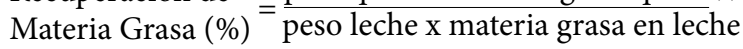

\section{Resultados}

\section{Rendimiento y parámetros fisicoquímicos de quesos}

En el Gráfico 1 se presentan los perfiles del rendimiento relativo al queso de referencia (100\%) obtenidos en la fabricación de los quesos en cada una de las condiciones del estudio, según el momento de agregado de TG y su concentración.

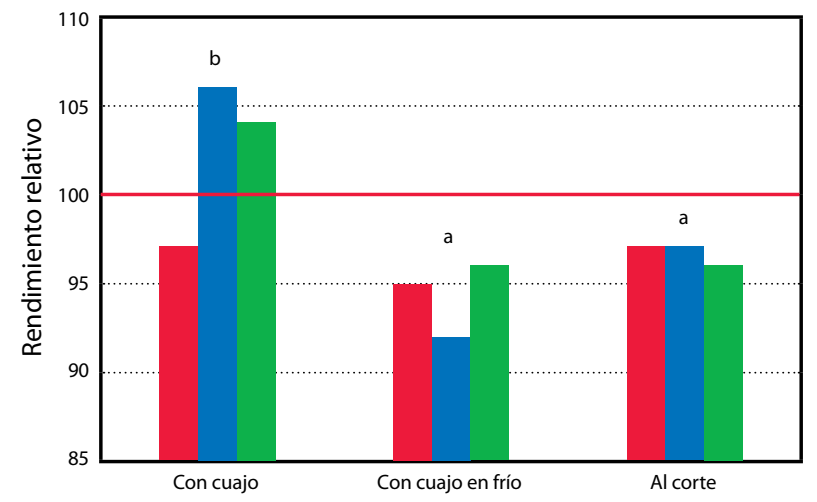

Momento de agregado de TG

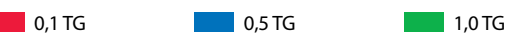

Gráfico 1. Rendimiento de la producción de quesos fabricados en las diferentes condiciones del estudio, relativo al queso referencia (100\%). Letras distintas indican medias con diferencia significativa $(p>0,05)$.

Del estudio del momento de agregado de la enzima (con el cuajo, con cuajo en frío y al corte) se observa que la única condición que mostró una diferencia significativa $(\mathrm{p}=0,005)$ en el rendimiento relativo fue el momento de agregado de la enzima, no importando la dosis de TG utilizada, ni encontrándose interacción entre los factores estudiados. El agregado de TG con el cuajo fue el que mostró el mayor rendimiento relativo del proceso.

No se encontraron diferencias significativas en la variación del peso de los quesos a lo largo del estudio.

Los aumentos de rendimiento encontrados fueron de un $6 \%$ y $4 \%$ respecto al queso referencia cuando la TG se incorporó junto con el cuajo en una concentración de 0,5 y $1,0 \mathrm{U}$ TG / g de proteína en la leche, respectivamente.

La recuperación relativa del extracto seco para los quesos a los que la TG se incorporó junto con el cuajo se muestra en el Gráfico 2. Se encontró diferencia significativa $(p=0,0009)$ en las concentraciones de TG utilizadas; los quesos fabricados con 0,5 y 1,0 U TG / g proteína fueron los que presentaron mayor recuperación relativa de extracto seco y no se encontraron diferencias a lo largo del tiempo.

En cuanto a la recuperación relativa de proteínas, no se hallaron diferencias respecto al momento de incorporación de la enzima a lo largo de todo el estudio. Al analizar la condición de agregado de la enzima con cuajo a lo largo del tiempo, se encontró que existe interacción entre el momento de agregado de la enzima y su concentración $(\mathrm{p}=0,0210)$. En la Tabla 1 se 
muestran las recuperaciones relativas de los quesos respecto al queso referencia. Se puede afirmar que a los 3 y 4 meses del estudio la concentración 1,0 U TG / g proteína da mayor recuperación relativa de proteínas que la concentración 0,1 U TG / g proteína.

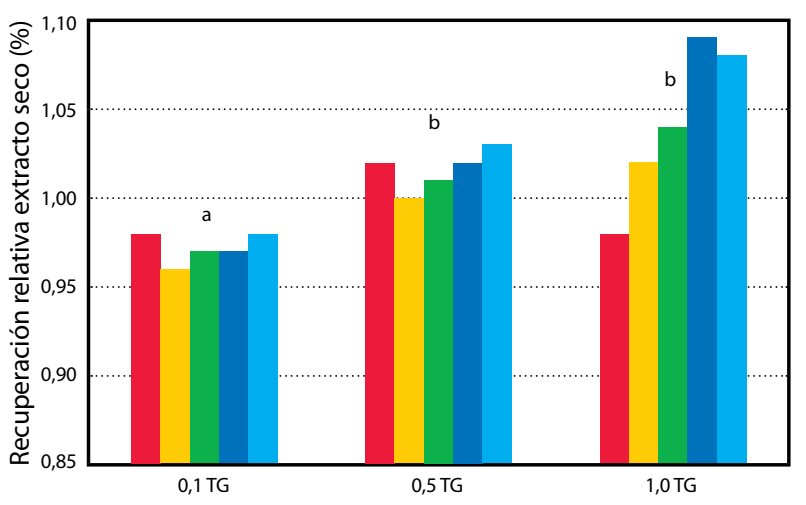

Concentración de TG (U)

MES 0

MES

MES 2

MES 3

Gráfico 2. Variación de la recuperación relativa del extracto seco a lo largo del tiempo del estudio para los quesos con TG incorporada con el cuajo. Letras distintas indican medias con diferencia significativa $(p>0,05)$.

\begin{tabular}{|c|c|c|c|c|c|}
\hline $\begin{array}{c}\text { U TG / g } \\
\text { proteína } \\
\text { agregadas }\end{array}$ & Mes 0 & Mes 1 & Mes 2 & Mes 3 & Mes 4 \\
\hline $0,1 \mathrm{TG}$ & $0,98 \mathrm{a}$ & $0,97 \mathrm{a}$ & $0,98 \mathrm{a}$ & $0,98 \mathrm{a}$ & $0,97 \mathrm{a}$ \\
$0,5 \mathrm{TG}$ & $0,99 \mathrm{ab}$ & $0,96 \mathrm{a}$ & $0,99 \mathrm{ab}$ & $0,98 \mathrm{a}$ & $0,99 \mathrm{abc}$ \\
$1,0 \mathrm{TG}$ & $0,96 \mathrm{a}$ & $1,02 \mathrm{abc}$ & $1,03 \mathrm{abc}$ & $1,08 \mathrm{c}$ & $1,08 \mathrm{c}$ \\
\hline
\end{tabular}

Tabla 1. Recuperación relativa de proteínas a lo largo del tiempo en los quesos con TG incorporada con cuajo en sus distintas concentraciones. Letras diferentes indican que los valores son significativamente diferentes al 5\% de confianza.

El estudio de la recuperación relativa de materia grasa mostró diferencias significativas ( $\mathrm{p}=0,003$ ) según el momento de agregado de la enzima, pero no se vio afectada por la concentración. Los momentos de agregado de la enzima al corte y con cuajo dieron mayores recuperaciones relativas de materia grasa que el momento en frío.

En el Gráfico 3 es posible apreciar la variación de la recuperación de materia grasa en los quesos fabricados con la incorporación de TG con cuajo. No se observaron diferencias significativas $(\mathrm{p}=0,0810)$ en esta recuperación al $5 \%$ de confianza. Al 10\% de confianza se puede afirmar que efectivamente existe diferencia entre la concentración 0,1 y las concentraciones 0,5 y 1,0U de TG/g proteína.

En cuanto a la humedad relativa solo se presentó una diferencia significativa según el momento de agregado de enzima a tiempo $0(p=0,008)$. En el tiempo 0 la humedad fue mayor al agregar la enzima junto con cuajo que al agregarla al corte. Se observó que al agregar la TG con cuajo las dosis utilizadas no presentan diferencias significativas al 5\% de confianza, aunque al $10 \%$ de significancia sí hay diferencias, siendo mayor la humedad con la dosis 0,5 de TG, tal como lo muestra el Gráfico 4.

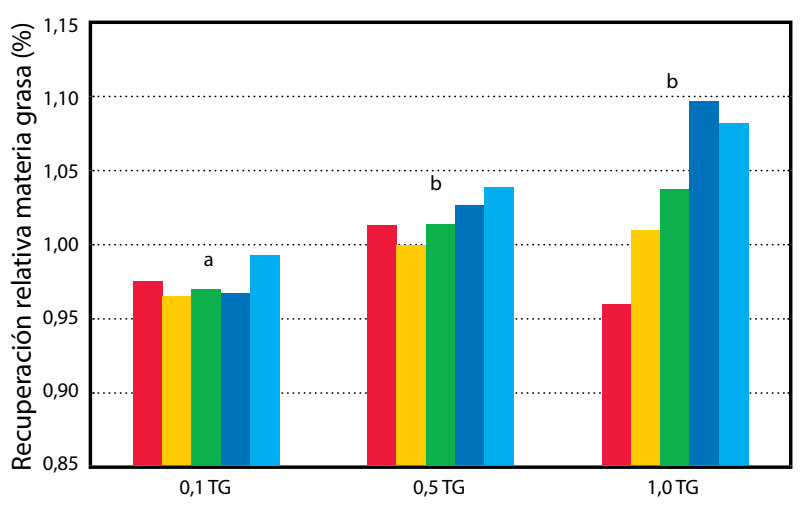

Concentración de TG (U)

\begin{tabular}{l|l|l|l|l} 
MES 0 & MES 1 & MES 2 & MES 3 & MES 4
\end{tabular}

Gráfico 3. Variación de la recuperación relativa de materia grasa a lo largo del tiempo para quesos fabricados con TG incorporada con el cuajo. Letras distintas indican medias con diferencia significativa $(\mathrm{p}>0,1)$.

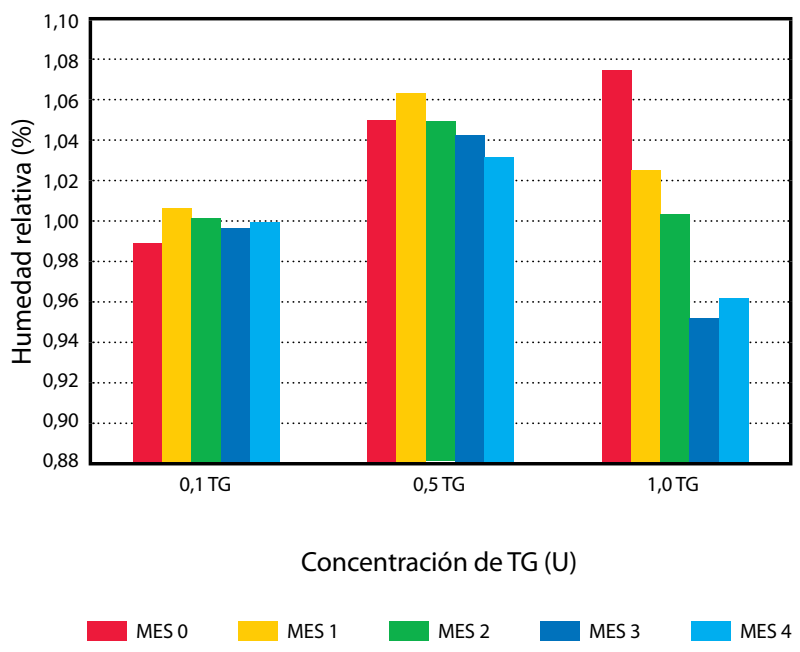

Gráfico 4. Variación de la humedad relativa de los quesos fabricados con TG incorporada con cuajo a lo largo del tiempo.

El Gráfico 5 muestra la variación del pH de los quesos fabricados con TG incorporada con cuajo. Se encontraron diferencias significativas según la concentración de enzima utilizada $(\mathrm{p}<0,001)$, obteniéndose valores de $\mathrm{pH}$ menores con el uso de 0,5 y 1,0 U TG/g proteína.

\section{Perfil de textura instrumental}

Respecto al análisis del perfil de textura, se analizaron los resultados de dureza, elasticidad y cohesividad.

En el Gráfico 6 se presentan los resultados de los análisis de dureza de los quesos con TG incorporada con cuajo a lo 


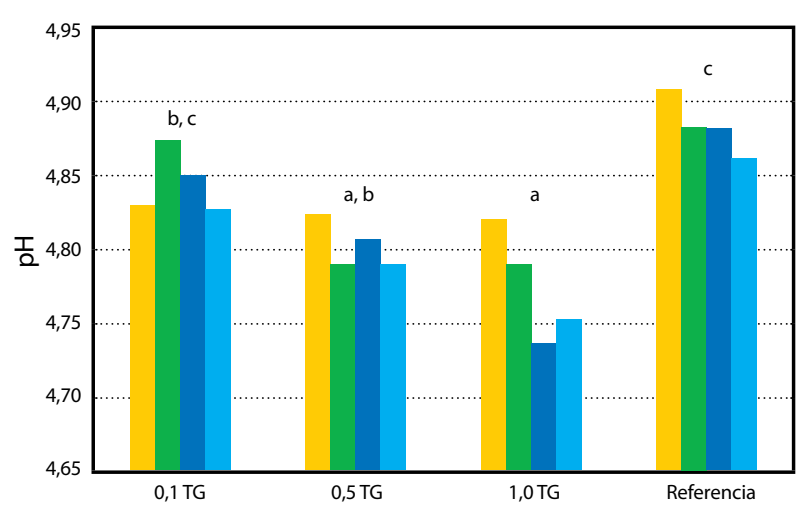

Concentración de TG (U)

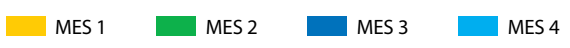

Gráfico 5. Variación del pH de los quesos fabricados con TG incorporada con cuajo a lo largo del tiempo del estudio. Letras distintas indican medias con diferencia significativa $(\mathrm{p}>0,05)$

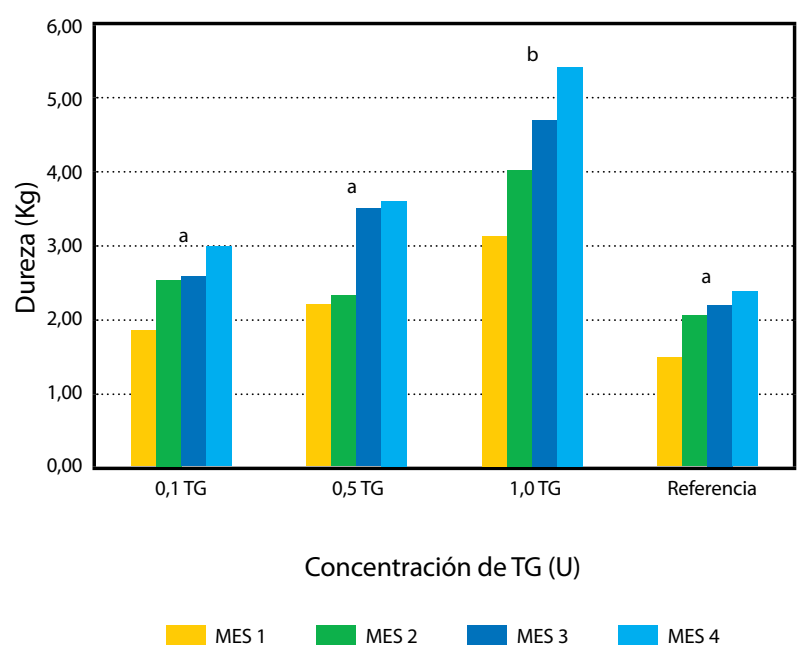

Gráfico 6. Dureza de los quesos fabricados incorporando la TG con cuajo y del queso referencia. Letras distintas indican medias con diferencia significativa $(p>0,05)$

largo del tiempo y de los quesos referencia correspondientes. Se puede observar que la concentración de TG afectó la dureza de los quesos fabricados a lo largo de todo el estudio, produciéndose los quesos más duros con 1,0 U de TG / g de proteína.

La elasticidad de los quesos (Gráfico 7) fue afectada significativamente tanto por la concentración de TG como por el momento de su incorporación. Los quesos con concentraciones de 0,5 y 1,0 U TG / g proteína son siempre más elásticos que los quesos con 0,1 U TG / g proteína.

Respecto a la cohesividad estudiada en la condición con cuajo a lo largo del tiempo, se observó que es mayor en los

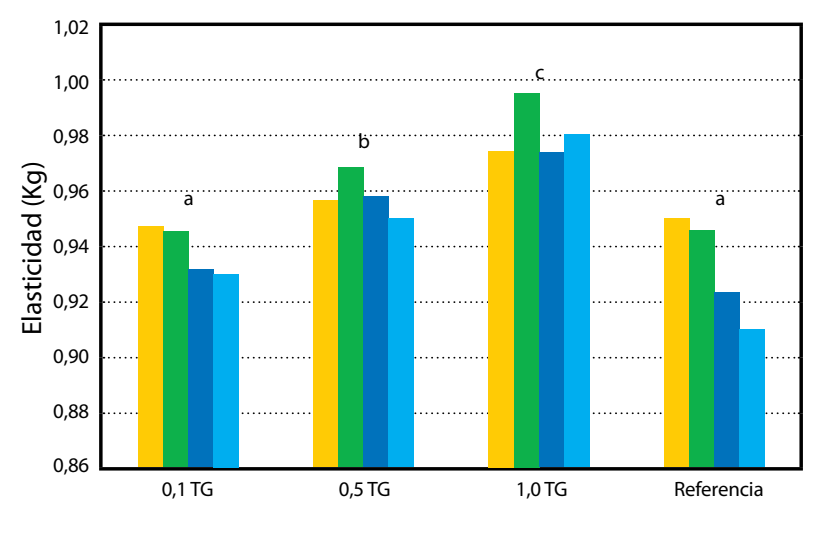

Concentración de TG (U)

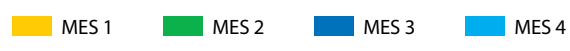

Gráfico 7. Elasticidad de los quesos fabricados incorporando la TG con cuajo y del queso referencia. Letras distintas indican medias con diferencia significativa $(\mathrm{p}>0,05)$.

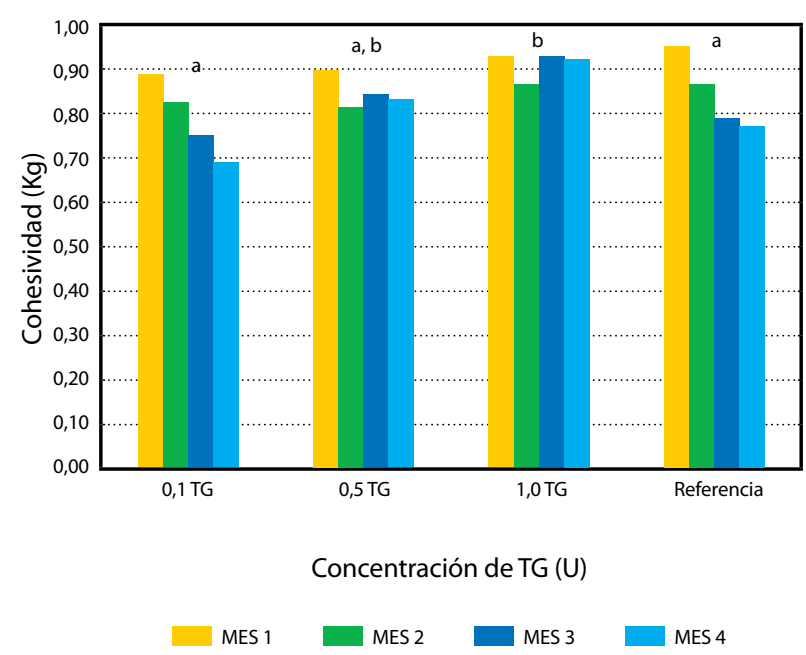

Gráfico 8. Cohesividad de los quesos fabricados incorporando la TG con cuajo y del queso referencia. Letras distintas indican medias con diferencia significativa $(\mathrm{p}>0,05)$

quesos fabricados con 1,0 U TG / g proteína que en el queso referencia (Gráfico 8).

\section{Perfil sensorial}

En el Gráfico 9 se presentan los resultados de la evaluación sensorial realizada por los jueces a los 30 días de maduración de los quesos elaborados con la TG incorporada con cuajo. Estos parámetros no experimentaron variaciones significativas a los 2, 3 y 4 meses del estudio, más allá de las propias del proceso de maduración del queso. 
Perfil sensorial a los 30 días de elaboración

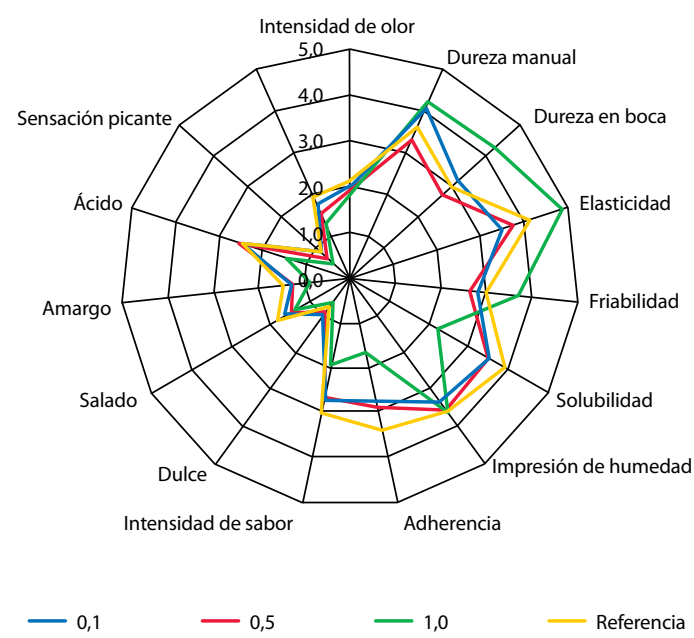

Gráfico 9. Resultados de los análisis sensoriales de los diferentes atributos estudiados para la condición con cuajo a los 30 días de maduración.

Desde el punto de vista sensorial se puede afirmar que a los 30 días de elaborados los quesos con 1,0 U TG / g proteína presentaron mayor dureza en boca y menor solubilidad $(\mathrm{p}<0,05)$ que los quesos fabricados con la menor concentración de enzima y que el queso de referencia. En cuanto a los cambios de los quesos con el tiempo a los 2, 3 y 4 meses del estudio no se hallaron más variaciones que las atribuibles al proceso de maduración de un queso.

\section{Discusión}

\section{Rendimiento y parámetros fisicoquímicos de quesos}

Hasta ahora se han realizado pocos estudios sobre la incorporación de TG en la fabricación de quesos. Özer et al. (2013) encontraron que la modificación de la matriz del queso debido a la reticulación de proteínas por TG es una herramienta eficaz para obtener un aumento de rendimiento en queso blanco aunque con características de textura modificadas. Se ha observado que el aumento en el rendimiento real de los quesos no se debe únicamente a la retención de más suero en el queso. Sayadia et al. (2013), en su estudio del tratamiento enzimático de la leche con TG, observaron un aumento en la relación humedad / proteína en el queso blanco iraní bajo en grasa.

Bönisch et al. (2008) estudiaron el efecto de la TG en la coagulación de la leche utilizando TG con adición de glutatión y constataron que al incorporar la enzima en la leche pre-incubada a distintos tiempos antes que el cuajo, la condición de agregado de TG junto con el cuajo fue la que presentó menor tiempo de coagulación, afectando el rendimiento del queso. La pre-incubación de la TG podría causar inhibición de la hidrólisis de la $\kappa$-caseína en la fase primaria de la coagulación.

A su vez, Özer et al. (2013) investigaron la adición de la TG a dos temperaturas: 30 y $34^{\circ} \mathrm{C}$, concluyendo que el uso de mayor temperatura provocó un mayor rendimiento en el queso blanco, aludiendo a que la temperatura presenta influencia en la cinética de la reorganización de las proteínas. En el presente estudio la utilización de $15^{\circ} \mathrm{C}$ (con cuajo en frío) presentó rendimientos menores al queso de referencia.

En base a los resultados de los estudios mencionados anteriormente, en este trabajo se constató que los mejores rendimientos en la producción de queso se obtuvieron con el agregado de la TG junto con el cuajo.

La mayor recuperación de extracto seco, encontrada al utilizar concentraciones de TG de 0,5 y 1,0 U TG / g proteína en esta investigación, podría deberse a la incorporación de proteína de suero en el queso, según lo demostrado por Özer et al. (2013), en el caso de queso blanco, y por Sayadia et al. (2013), en queso blanco iraní bajo en grasa.

En base al Gráfico 1 y los resultados de contenido de humedad y materia grasa, podría afirmarse que la mayor humedad y recuperación relativa de materia grasa cuando se agrega TG con cuajo inducen el mayor rendimiento debido a una mayor retención de agua y materia grasa en la red proteica. Aaltonen et al. (2014) demostraron una mayor incorporación de agua en queso Edam estandarizado con proteínas de suero.

\section{Perfil de textura instrumental}

Sayadia et al. (2013) hallaron que la dureza del queso blanco iraní aumenta con la concentración de TG utilizada en su fabricación, tal como se demostró con este estudio para el queso Dambo tipo barra, correlación similar a la hallada por Özer et al. (2013). Estos autores atribuyen a que la función principal de TG en el queso es formar enlaces isopeptídicos, lo que conduce a una red de gel con menores agregados y tamaños de poros, resultando en una red más restringida, por lo que se prevé que la dureza sea debido a una mayor cantidad de puntos de contacto entre las proteínas de la matriz del queso.

En lo referente a la elasticidad de los quesos, Özer et al. (2013) determinaron que los quesos tratados con TG mantienen la elasticidad más estable durante la maduración, comparados con los quesos que no se elaboran con TG. En este estudio esto se dio para los quesos fabricados con la mayor concentración de TG adicionada junto con el cuajo (0,5 y 1,0 U TG / g proteína).

Se constató que los quesos fabricados con 0,5 y $1,0 \mathrm{U}$ TG / g proteína son los que presentaron mayores valores de cohesividad. En el trabajo de Özer et al. (2013) se demostró que la cohesividad no presenta diferencias significativas con el tiempo, sin embargo, se hallaron valores más altos en el queso blanco salado tratado con TG respecto a la referencia. Esto se justifica porque tanto el número como la fuerza de los enlaces de la proteína en los quesos tratados con enzimas son afectados por la TG.

\section{Perfil sensorial}

Los quesos fabricados con 1,0 U TG / g proteína incorporada junto con el cuajo mostraron cambios significativos en su textura, ya que presentaron mayor dureza, elasticidad y cohesividad, y también en su sabor, pues tuvieron menor intensidad de sabor, menor sabor ácido y menor sensación picante. Esto último puede deberse a que al tener más enzima los quesos fueron menos solubles, provocando una menor movilidad de los sabores en la lengua, lo que llevó a una detección menor. 


\section{Conclusiones}

El mayor rendimiento quesero se dio al incorporar la TG junto con cuajo. Con los estudios específicos que se hicieron de esa condición, se constató que al incorporar 0,5 y 1,0 U TG / g de proteína el rendimiento aumentó un 6 y un 4\%, respectivamente, en relación al queso Dambo barra tradicional sin enzima. A efectos prácticos, en la fabricación del queso Dambo se entiende que la condición con cuajo es la más fácil de adecuar a las condiciones que poseen las fábricas, porque se puede hacer con la infraestructura de una quesería tradicional y en las mismas condiciones de proceso, sin requerir mayor know-how, equipamiento, entrenamiento adicional del personal, y simplemente incorporando un ingrediente más en la fabricación.

Los quesos con TG incorporada con cuajo como coadyuvante en su fabricación mantienen las características propias del queso Dambo tipo barra con una mayor dureza que el queso tradicional, lo cual es un atributo deseable para obtener mejores características de feteabilidad.

\section{Reconocimientos}

Los autores agradecen la iniciativa, el apoyo permanente y la colaboración de los representantes de Ajinomotodo Brasil Indústria e Comércio de Alimentos Ltda., especialmente a Claudio Pagani, Thiago Granado Franco, Ryusuke Kojima y Andrea Valezin.

También agradecen el apoyo analítico brindado por el Departamento de Productos Agropecuarios de la Gerencia de Análisis, Ensayos y Metrología del LATU, en particular a Jacqueline Cea, Karina Salvo y María Victoria Figueredo.

\section{Referencias}

Aaltonen, T., Huumonen, I. y Myllärinen, P., 2014. Controlled transglutaminase treatment in Edam cheese-making. En: International Dairy Journal. Artículo en impresión, 38(2), pp.179-182.

AOAC International, 2005. Official methods of analysis of AOAC International. 18a ed. Gaithersburg: AOAC. Official Method 925.22.

Bönisch, M.P., Heidebach, T.C. y Kulozik, U., 2008. Influence of transglutaminase protein cross-linking of the rennet coagulation of casein micelles. En: Food Hydrocolloids, 22, pp.288-297.

Bönisch, M.P., Huss, M., Weitl, K. y Kulozik, U., 2007. Transglutaminase cross-linking of milk proteins and impact on yoghurt gel properties. En: International Dairy Journal, 17, pp.1360-1371.

Cozzolino, A., Di Pierro, P., Mariniello, L., Sorrentino, A., Masi, P. y Porta, R., 2003. Incorporation of whey proteins into cheese curd by transglutaminase. En: Biotechnology Applied Biochemestry, 38, pp.289-295.

International Organization for Standardization, 2012. ISO/TS 22113 (IDF RM 204): Milk and milk products. Determination of the titratable acidity of milk fat. Ginebra: ISO.

International Organization for Standardization, 2010. ISO 1211 (IDF 1): Milk - Determination of fat content. Gravimetric method (Reference method). Ginebra: ISO.
International Organization for Standardization, 2010. ISO 6731 (IDF 21): Milk, cream and evaporated milk. Determination of total solids content (Reference method). Ginebra: ISO.

International Organization for Standardization, 2009. ISO 22935-1 (IDF 99-1): Milk and milk products. Sensory analysis. Part 1: General guidance for the recruitment, selection, training and monitoring of assessors. Ginebra: ISO.

International Organization for Standardization, 2007. ISO 8589: Sensory analysis: general guidance for the design of test rooms. Ginebra: ISO.

International Organization for Standardization, 2004. ISO 1735 (IDF 005): Cheese and processed cheese products. Determination of fat content. Gravimetric method (Reference method). Ginebra: ISO.

International Organization for Standardization, 2004. ISO 5534 (IDF 4): Cheese and processed cheese. Determination of the total solids content (Reference method). Ginebra: ISO.

International Organization for Standardization, 2001. ISO 8968 (IDF 20): Milk. Determination of nitrogen content (Kjeldahl method). Ginebra: ISO.

Jaros, D., Jacob, M., Otto, C. y Rohm H., 2010. Excessive crosslinking of caseins by microbial transglutaminase and its impact on physical properties of acidified milk gels. En: International Dairy Journal, 20, pp.321-327.

Jaros, D., Heidig, C.yRohm, H., 2007. Enzymatic modification through microbial transglutaminase enhances the viscosity of stirred yogurt. En: Journal of Texture Studies, 38, pp.179-198.

Kuraishi, C.,Yamazaki, K. y Susa, Y., 2001. Transglutaminase: its utilization in the food industry. En: Food Reviews International, 17(2), pp.221-246.

Lavanchy, P., Berodier, F., Zannoni, M., Noël, Y., Adamo, C., Squella, J. y Herrero, L., 1993. L' évaluation sensorielle de la texture des fromages à pâte dure ou semidure. Étude interlaboratoires. En : Lebensm. Wiss. Techno., 26, pp.5-68.

MERCOSUR, 1994. Resolución GMC/RES 79/94, del 4 de noviembre de 1994. Identidad y calidad de quesos. Brasilia: Mercosur

Ministerio de Ganadería Agricultura y Pesca, 2013. Estadísticas del sector lácteo 2011. Montevideo: DIEA.

Montero, H., Aranibar, G., Cañameras, C., y Castañeda, R., 2005. Metodologías para la caracterización sensorial de quesos argentinos. En: Jornadas de Análisis Sensorial (JASLIS 2005), (Buenos Aires 6-8 de septiembre de 2005). Buenos Aires: [s.n.].

Özer, B., Adnan Hayaloglu, A., Yaman, H., Gürsoy, A. y Sener, L., 2013. Simultaneous use of transglutaminase and rennet in white-brined cheese production. En: International Dairy Journal, 33(2), pp.129-134.

Özer, B., Guyot, C. y Kulozik, H., 2012. Simultaneous use of transglutaminase and rennet in milk coagulation: Effect of initial milk $\mathrm{pH}$ and renneting temperature. En: International Dairy Journal, 24, pp.1-7.

Özrenk, E., 2006. The use of transglutaminase in dairy products. En: International Journal of Dairy Technology, 59, pp.1-7.

Sayadia, A., Madadloub, A. y Khosrowshahia, A., 2013. Enzymatic cross linking of whey proteins in low fat Iranian white cheese. En: International Dairy Journal, 29(2), pp.88-92. 